\title{
Unhealthy Drinking Patterns in Older Adults: Prevalence and Associated Characteristics
}

\author{
Elizabeth L. Merrick, PhD, * Constance M. Horgan, ScD, , Dominic Hodgkin, PhD, * \\ Deborah W. Garnick, ScD, ${ }^{*}$ Susan F. Houghton, BS, ${ }^{*}$ Lee Panas, MS, ${ }^{*}$ Richard Saitz, MD, MPH, ${ }^{\dagger \neq \mathcal{S}}$ \\ and Frederic C. Blow, PhD"\#
}

\begin{abstract}
OBJECTIVES: To examine the prevalence of unhealthy drinking patterns in community-dwelling older adults and its association with sociodemographic and health characteristics.
\end{abstract}

DESIGN: Cross-sectional analysis of nationally representative survey data.

SETTING: The data source was the 2003 Access to Care file of the Medicare Current Beneficiary Survey, which represents the continuously enrolled Medicare population.

PARTICIPANTS: Community-dwelling Medicare beneficiaries aged 65 and older $(\mathrm{N}=12,413)$.

MEASUREMENTS: The prevalence of unhealthy alcohol use by older adults defined in relation to two parameters of recommended limits: monthly use exceeding 30 drinks per typical month and "heavy episodic" drinking of four or more drinks in any single day during a typical month in the previous year. Sociodemographic and health status variables were also included.

RESULTS: Nine percent of elderly Medicare beneficiaries reported unhealthy drinking, with higher prevalence in men $(16 \%)$ than women $(4 \%)$. In logistic regression analyses with the full sample, higher education and income; better health status; male sex; younger age; smoking; being white; and being divorced, separated, or single were associated with higher likelihood of unhealthy drinking. Among drinkers, in addition to sociodemographic variables, selfreported depressive symptoms were positively associated with unhealthy drinking. Among unhealthy drinkers, race

From the *Institute for Behavioral Health, Heller School for Social Policy and Management, Brandeis University, Waltham, Massachusetts; ${ }^{\dagger}$ Clinical Addiction Research and Education Unit, Section of General Internal Medicine, Boston Medical Center, Boston, Massachusetts; ${ }^{\ddagger}$ Youth Alcohol Prevention Center and ${ }^{\mathrm{S}}$ Department of Epidemiology, Boston University School of Public Health, Boston, Massachusetts; "Department of Psychiatry, University of Michigan, Ann Arbor, Michigan; ${ }^{\#}$ Department of Veterans Affairs, Health Services Research and Development, Serious Mental Illness Treatment Research and Evaluation Center, Ann Arbor, Michigan.

Address correspondence to Elizabeth L. Merrick, PhD, Senior Scientist, Institute for Behavioral Health, Heller School for Social Policy and Management, Brandeis University, Mail stop 035, 415 South St., Waltham, MA 02454. E-mail: merrick@brandeis.edu

DOI: 10.1111/j.1532-5415.2007.01539.x and ethnicity variables were associated with likelihood of heavy episodic drinking.

CONCLUSION: Almost one in 10 elderly Medicare beneficiaries report exceeding recommended drinking limits. Several distinct unhealthy drinking patterns were identified and associated with sociodemographic and health characteristics, suggesting the value of additional targeted approaches within the context of universal screening to reduce alcohol misuse by older adults. J Am Geriatr Soc 56:214-223, 2008.

Key words: alcohol misuse; elderly; prevalence

$\mathrm{U}$ nhealthy use of alcohol by older adults is a serious problem and is underidentified and undertreated. ${ }^{1}$ Unhealthy alcohol use has been defined as encompassing risky use, problem drinking, and alcohol disorders including abuse and dependence. ${ }^{2}$ The costs in dollars and health effects arising from older adults' unhealthy alcohol use are substantial, and the magnitude is likely to grow as the large baby-boom generation ages. Elderly people are hospitalized for conditions associated with alcohol at approximately the same rate as for myocardial infarction. ${ }^{3}$ Alcohol use can result in falls leading to hip fracture, a leading cause of death in this group. ${ }^{4}$ Older adults' higher sensitivity and poorer ability to metabolize alcohol contribute to higher risk at a given level of use. ${ }^{4}$ Furthermore, alcohol can exacerbate medical disorders that are common in elderly people, including congestive heart failure and hypertension. ${ }^{4}$ The potential for adverse interactions between alcohol and medications adds to the risk. These risks must be taken into account along with the more highly publicized findings of health benefits associated with moderate drinking $^{5-7}$ in determining appropriate recommendations for older adults' alcohol consumption.

Recommended drinking limits are lower than for younger people. National Institute on Alcohol Abuse and Alcoholism (NIAAA) and American Geriatrics Society (AGS) clinical guidelines currently define risky drinking amounts for people aged 65 and older as more than seven 
drinks per week or more than three drinks on any single day. ${ }^{8,9}$ Among elderly people, exceeding these limits is associated with significant interpersonal and functioning problems. ${ }^{10}$ Substance Abuse and Mental Health Services Administration recommendations are similar but note that older women should drink somewhat less than men and set the single-occasion threshold at no more than two drinks. ${ }^{1}$ For those with health conditions exacerbated by alcohol or using medications with which alcohol is contraindicated, even lower limits or abstinence are recommended.

Prevalence estimates of unhealthy drinking among older adults range from $1 \%$ to $15 \%$ and higher, ${ }^{11,12}$ partly due to widely varying definitions (formal alcohol disorders, drinking exceeding varying cutoffs, drinking when contraindicated because of comorbidities or medication use). Men aged 65 and older have a higher prevalence of unhealthy drinking than women, ranging from $10 \%$ to $15 \%$ versus $2 \%$ to $5 \% .{ }^{13}$ In three nationally representative surveys, $9 \%$ to $10 \%$ of men and $2 \%$ to $3 \%$ of women aged 65 and older were found to be heavy drinkers (defined as $>1$ drink per day). ${ }^{14}$ The 2005 National Survey on Drug Use and Health found that $8.3 \%$ of people aged 65 and older had binge use ( $\geq 5$ drinks on the same occasion at least once in the previous 30 days) and $1.7 \%$ were heavy drinkers ( $\geq 5$ drinks on the same occasion on $\geq 5$ days in the previous 30 days). ${ }^{15}$ Another study found a $4 \%$ prevalence of binge drinking ( $\geq 5$ drinks on at least one occasion in the previous month) in persons aged 55 and older. ${ }^{16}$ A large study of older primary care patients found that $4.1 \%$ were at-risk drinkers (defined as $8-14$ drinks per week) and $4.5 \%$ were heavy drinkers ( $\geq 14$ drinks per week) or binge drinkers ( $\geq 4$ drinks in single day over the previous 3 months). ${ }^{17}$ Alcohol dependence in elderly people has been estimated at less than $4 \%{ }^{18}$ or even less than $1 \%,{ }^{19}$ whereas alcohol abuse meeting Diagnostic and Statistical Manual of Mental Disorders, Fourth Edition, diagnostic criteria is estimated at $2 \%$ for men and less than $1 \%$ for women. ${ }^{19}$

Factors found to be associated with a higher likelihood of unhealthy drinking in older adults include male sex, younger age, more-active lifestyle, better health and functional status, and smoking. ${ }^{20}$ Studies have variously found that living alone for men or with a spouse for men and women ${ }^{20}$ and living alone or being divorced ${ }^{21}$ or unmarried predicts unhealthy drinking. ${ }^{22}$ There is evidence of higher unhealthy drinking prevalence among elderly non-Hispanic whites. ${ }^{14}$ Although depression can be a result of drinking in elderly people, ${ }^{23,24}$ findings regarding correlation with unhealthy drinking amounts are mixed. ${ }^{17,20,25,26}$

Identification of older adults who are drinking risky amounts is essential for appropriate and timely clinical intervention. ${ }^{27}$ For clinicians in healthcare settings in which elderly people are treated, understanding alcohol consumption patterns and patient characteristics associated with unhealthy alcohol use is an important adjunct to formal screening tools. Given widespread underrecognition of unhealthy alcohol use, being especially alert to risk factors may assist in detection. Clinicians may be less likely to suspect unhealthy alcohol use in older adults, ${ }^{1}$ and physicians are less likely to identify alcohol disorders in patients with higher income or education and in women. ${ }^{28}$ Analysis of drinking patterns in the overall population may suggest needed public education interventions at broader levels.
Although prior studies have examined unhealthy drinking in older adults, additional research is needed. ${ }^{10,23} \mathrm{Few}$ previous studies have focused on multiple patterns of unhealthy drinking directly in relation to current guidelines using nationally representative data. This study contributes by developing up-to-date, nationally representative prevalence estimates of unhealthy drinking by examining consumption of risky alcohol amounts by the elderly Medicare population in relation to current guidelines. Variation in unhealthy alcohol consumption patterns was also examined in the context of a wide range of sociodemographic and health characteristics, including some such as depression and functional status that are not always included in analyses.

\section{METHODS}

\section{Sample}

The data source was the 2003 Access to Care file of the Medicare Current Beneficiary Survey (MCBS), which is designed to represent the continuously enrolled Medicare population. ${ }^{29}$ The MCBS is an ongoing survey of a representative national sample of the Medicare population by the Centers for Medicare and Medicaid Services. It contains items regarding utilization, payment, health status, functioning, and health behaviors. The 2003 MCBS included items addressing alcohol consumption. ${ }^{30}$

Beneficiaries sampled from Medicare enrollment files (or proxies) are interviewed three times a year using inperson, computer-assisted interviewing. There is a 4-year rotating panel design. Beneficiaries were randomly selected according to age strata from a nationally representative set of 107 geographic primary sampling units, with oversampling of the disabled (aged $<65$ ) and those aged 85 and older. Normalized sampling weights were assigned to represent the population.

The 2003 MCBS Access to Care sample consisted of 16,003 beneficiaries; 3,520 subjects younger than 65 or residing in a facility were excluded. Respondents with missing data on alcohol survey items were also excluded $(n=70$; $<1 \%$ of eligible sample). The analytical sample consisted of 12,413 community-dwelling, full-year Medicare beneficiaries aged 65 and older.

\section{Alcohol Consumption Variables}

The 2003 MCBS survey included three items regarding alcohol consumption. ${ }^{30}$ Quantity and frequency were ascertained by asking, "Please think about a typical month in the past year. On how many days did you drink any type of alcoholic beverage? On those days that you drank alcohol, how many drinks did you have?" Heavy episodic drinking was assessed by asking, "Please think about a typical month in the past year. On how many days did you have four or more drinks in a single day?" Alcoholic beverages were described as including "liquor such as whiskey or gin, mixed drinks, wine, beer, and any other type of alcoholic beverage."

To assess unhealthy drinking in terms of consuming risky amounts of alcohol (regardless of whether alcohol problems or disorders were present), alcohol measures were defined reflecting parameters of the NIAAA and AGS 
guidelines. First, to be consistent with the weekly guideline, unhealthy drinking was defined as exceeding 30 drinks per typical month. Second, a single-day or "heavy episodic drinking" variable was constructed indicating whether an individual reported drinking four or more drinks in any single day during a typical month in the previous year. Exceeding either limit defined unhealthy drinking.

Respondents were initially categorized as nondrinkers, within-guidelines drinkers (not exceeding the 30-drink monthly limit and not exceeding 3 drinks on any day), drinkers who exceeded the monthly 30-drink limit only, drinkers who exceeded the single-day drinking limit only (drank $>3$ drinks on any one day but did not exceed the monthly limit), and drinkers who exceeded both the monthly and single-day limits. Dichotomous dependent variables were then created for multivariate analysis. Unhealthy drinkers were drinkers who reported any type of risky drinking, exceeding either monthly or single-day limits. "Heavy episodic drinkers" exceeded the three-drink limit on at least 1 day during a typical month in the previous year. Dual-risk drinkers exceeded both the 30-drink monthly limit and the single-day threshold. This categorization was used to identify distinct patterns of unhealthy drinking and to facilitate the identification of factors associated with each.

\section{Independent Variables Sociodemographics}

Variables were included for sex, race (white, black, other), Hispanic ethnicity, annual income $(<\$ 25,000 ; \$ 25,000-$ $40,000 ;>\$ 40,000)$, age $(65-70 ; 71-80 ; \geq 81)$, education (less than high school diploma; high school graduate; some college, technical, vocational or business school; bachelors degree or higher), a dichotomous variable indicating residence in a metropolitan area, and marital status (married; widowed; divorced, separated, or single at the time of survey). Living arrangement was highly correlated with marital status, so it was not included.

\section{Health Status and Functioning}

Self-reported health status compared with others the same age was categorized as excellent, good or very good, and poor or fair. The Katz Index of independence in activities of daily living (ADLs) was used to construct a modified measure of functional independence. ${ }^{31}$ Respondents were asked whether they had any difficulty bathing, dressing, transferring, toileting, or feeding and whether they experienced urinary incontinence. ${ }^{30}$ Positive responses on items asking whether respondents used special equipment to accomplish the ADL or had someone usually stay nearby in case they needed help with bathing, dressing, eating, transferring, or toileting, thus reflecting a slightly broader capture of difficulty indicators, were also included. The resulting variable or modified Katz score reflects a 7-position scale (0-6) of the number of self-reported independent ADLs. A score of 6 indicates full function, 4 indicates moderate functional impairment, and 2 or less indicates significant functional impairment. ${ }^{31}$

\section{Behavioral Health}

Respondents were asked, "In the past 12 months, how much of the time did you feel sad, blue, or depressed?
Would you say you were sad or depressed all of the time, most of the time, some of the time, a little of the time, or none of the time?" ${ }^{30}$ Beneficiaries who reported depressive symptoms all or most of the time were categorized as experiencing chronic depressive symptoms. Current smoking status was also included.

\section{Statistical Analysis}

Results presented are weighted estimates designed to represent the continuously enrolled elderly Medicare population at the time of the 2003 MCBS survey. In addition to global chi-square tests for overall differences, pairwise chisquare tests were used to determine significance of differences in specific drinking pattern according to sociodemographic, health status and other factors. The Bonferonni correction was applied to adjust significance level for multiple comparisons.

Three sets of logistic regression analyses were conducted to model various unhealthy drinking patterns as a function of sociodemographic, health, and functional status characteristics. In one set, any unhealthy drinking (exceeding monthly 30-drink limit or single-day limit) was analyzed. In the second set, heavy episodic drinking (exceeding the single-day threshold) was modeled. In a third set, dualrisk drinking (exceeding monthly and single-day thresholds) was modeled to identify factors associated with this drinking pattern, which could constitute a particularly high-risk category. This was done using the full sample, the subsample of drinkers, and the subsample of unhealthy drinkers to examine how factors might vary depending on the target population represented, thus assisting in recognition of particularly high risk within population and drinker groups. Finally, ordinary least squares regression was conducted for the drinker subsample, with a continuous dependent variable measuring number of days of heavy episodic drinking in a typical month. If there was a discrepancy between responses on the heavy episodic drinking item and the drinking days and drinks per day items on the MCBS, the higher of the two self-reports was used, because underreporting is believed to be more likely than overreporting. Ordinary least squares regression results are reported, but Poisson regression modeling had qualitatively similar results.

Because of the sampling design, using procedures that assumed equal probability of selection would likely lead to underestimating standard errors. The SVY:LOGIT procedure of the statistical package Stata (Stata Corp., College Station, TX) was used to take into account the complex sample design. This allowed for more-accurate estimation of standard errors and more-valid confidence intervals and significance tests. Stata is one of several statistical software programs available to analyze data based on complex survey designs, which require "design based" analysis. ${ }^{32,33}$

\section{RESULTS}

\section{Sample Description}

Sample characteristics are presented in Table 1. The sample, after weighting, reflected a predominantly white $(87 \%)$ non-Hispanic $(93 \%)$ population living in a metropolitan area $(77 \%)$. More than half were female $(57 \%)$. Thirty 
Table 1. Sample Description: 2003 Medicare Current Beneficiary Survey Access to Care Module, CommunityDwelling Persons Aged 65 and Older

\begin{tabular}{|c|c|c|c|}
\hline Characteristic & $\begin{array}{c}\text { Unweighted } \\
\text { N }\end{array}$ & $\begin{array}{l}\text { Weighted } \\
\text { N }\end{array}$ & $\begin{array}{l}\text { Weighted } \\
\text { Percentage }\end{array}$ \\
\hline Total & 12,413 & $31,365,267$ & 100.0 \\
\hline \multicolumn{4}{|l|}{ Sex } \\
\hline Female & 7,078 & $17,912,983$ & 57.0 \\
\hline Male & 5,335 & $13,452,284$ & 43.0 \\
\hline \multicolumn{4}{|l|}{ Age } \\
\hline $65-70$ & 3,331 & $9,425,123$ & 30.0 \\
\hline $71-80$ & 5,163 & $14,564,591$ & 46.0 \\
\hline$\geq 81$ & 3,919 & $7,375,553$ & 24.0 \\
\hline \multicolumn{4}{|l|}{ Ethnicity } \\
\hline Hispanic & 892 & $2,267,080$ & 7.0 \\
\hline Non-Hispanic & 11,508 & $29,053,508$ & 93.0 \\
\hline \multicolumn{4}{|l|}{ Race } \\
\hline White & 10,862 & $27,318,350$ & 87.3 \\
\hline Black & 1,016 & $2,594,439$ & 8.3 \\
\hline Other & 509 & $1,389,583$ & 4.4 \\
\hline \multicolumn{4}{|l|}{ Education } \\
\hline Less than high school diploma & 3,827 & $9,159,886$ & 29.0 \\
\hline High school graduate & 3,663 & $9,404,390$ & 30.0 \\
\hline $\begin{array}{l}\text { Some college, vocational, } \\
\text { technical, or business school }\end{array}$ & 2,917 & $7,526,659$ & 24.0 \\
\hline Bachelors degree or higher & 1,961 & $5,173,902$ & 17.0 \\
\hline \multicolumn{4}{|l|}{ Income, \$ } \\
\hline$<25,000$ & 7,316 & $17,726,147$ & 58.0 \\
\hline $25,000-40,000$ & 2,568 & $6,734,971$ & 22.0 \\
\hline$>40,000$ & 2,190 & $5,938,385$ & 20.0 \\
\hline \multicolumn{4}{|l|}{ Marital status } \\
\hline Married & 6,593 & $17,415,159$ & 55.5 \\
\hline Widowed & 4,476 & $10,306,654$ & 32.9 \\
\hline Divorced, separated, or single & 1,339 & $3,634,225$ & 11.6 \\
\hline Living in metropolitan area & 9,206 & $24,188,387$ & 77.0 \\
\hline \multicolumn{4}{|l|}{ Self-reported health status } \\
\hline Excellent & 2,044 & $5,258,510$ & 17.0 \\
\hline Good to very good & 7,490 & $19,067,389$ & 61.0 \\
\hline Poor to fair & 2,836 & $6,938,637$ & 22.0 \\
\hline \multicolumn{4}{|l|}{ Self-reported functional status } \\
\hline $\begin{array}{l}\text { Mean number of } \\
\text { independent ADLs }\end{array}$ & & & 5.2 \\
\hline \multicolumn{4}{|l|}{ Number of ADLs independent in } \\
\hline $0-2$ & 720 & $1,643,829$ & 5.3 \\
\hline 3 or 4 & 1,344 & $3,110,629$ & 10.0 \\
\hline 5 & 2,973 & $7,452,546$ & 24.0 \\
\hline 6 & 7,254 & $18,870,551$ & 60.7 \\
\hline $\begin{array}{l}\text { Depressed all or most } \\
\text { of previous year }\end{array}$ & 680 & $1,680,447$ & 5.4 \\
\hline Current smoker & 1,287 & $3,439,819$ & 11.0 \\
\hline
\end{tabular}

Note: Respondents with missing data on alcohol survey items were excluded from the sample ( $\mathrm{n}=70,<1 \%$ unweighted of otherwise eligible respondents).

Distribution shown reflects nonmissing cases within each variable (missing $<2.7 \%$ ).

$\mathrm{ADL}=$ activity of daily living. percent were aged 65 to $70,46 \%$ were aged 71 to 80 , and $24 \%$ were aged 81 and older. More than half $(58 \%)$ had an annual income of less than $\$ 25,000$ and had received no more than a high school education $(59 \%)$. More than half were married $(56 \%)$ at the time of the survey, and most reported good or very good health $(61 \%)$ and full independence in activities of daily living $(61 \%)$. Eleven percent were current smokers, and $5 \%$ reported feeling sad, blue, or depressed for all or most of the previous year.

\section{Bivariate Findings}

The weighted prevalence of specific alcohol consumption patterns, broken out according to sociodemographic and health characteristics, is presented in Table 2. Two thirds of elderly people reported no alcohol consumption during a typical month in the previous year $(65 \%), 26 \%$ reported alcohol consumption consistent with guidelines, and $9 \%$ reported consuming alcohol in ways that exceeded the monthly or single-day limits. Approximately $3.7 \%$ exceeded the 30 -drink monthly limit only, $2.2 \%$ exceeded the single-day limit of three drinks only, and 3.1\% exceeded both limits. Thus, of those drinking risky amounts, $58.9 \%$ exceeded the single-day limit $(5.3 \%$ of the total $9 \%$ of unhealthy drinkers). Of those with heavy episodic drinking, the mean number of heavy episodic drinking days in a typical month was 9 (standard error 0.5 ), and the median was 4 (data not shown).

All sociodemographic, health, and functional status variables were significantly associated with drinking pattern in the full sample and many pairwise difference were also significant (Table 2). Women were more likely to be nondrinkers and less likely to report unhealthy consumption ( $4 \%$ of women and $16 \%$ of men exceeded either the monthly or episodic threshold, $P<.01$ ). Those aged 81 and older were most likely to be nondrinkers and least likely to report unhealthy drinking $(4.9 \%$, vs $13 \%$ for those aged $65-70$ and $8.7 \%$ for those aged $71-80, P<.01)$. Unhealthy drinking was more prevalent among whites $(P<.01)$, whereas higher levels of education and income were associated with higher prevalence of drinking within guidelines and unhealthy drinking $(P<.01)$. Being widowed was associated with lower prevalence of unhealthy drinking $(P<.01)$. The percentage of persons exceeding the monthly or single-day parameters declined with self-reported health status $(P<.01)$. Those who reported depressive symptoms for most or all of the previous year were less likely to be unhealthy drinkers $(P<.01)$. Better functional status was positively associated with unhealthy drinking $(11 \%$ in the highest-functioning category, vs $3 \%$ in the lowest, $P<.01$ ).

All sociodemographic, health, and functional status variables were significantly associated with drinking patterns overall in the unhealthy drinker sample (all global chi-square tests, $P<.01)$. Furthermore, many pairwise comparisons of prevalence of specific unhealthy drinking patterns yielded significant relationships (Table 2). For example, $6.2 \%$ of men exceeded both monthly and single-day limits, compared with $0.7 \%$ of women $(P<.01)$. College graduates had a higher prevalence of every specific unhealthy drinking pattern and were especially more likely to drink over monthly limits only $(8 \%$, vs $1.7 \%$ for those without a high school diploma, $P<.01$ ). Income followed a similar pattern, with higher 
Table 2. Prevalence of Drinking Patterns According to Sociodemographic and Health Characteristics: 2003 Medicare Current Beneficiary Survey Access to Care Module, Community-Dwelling Persons Aged 65 and Older

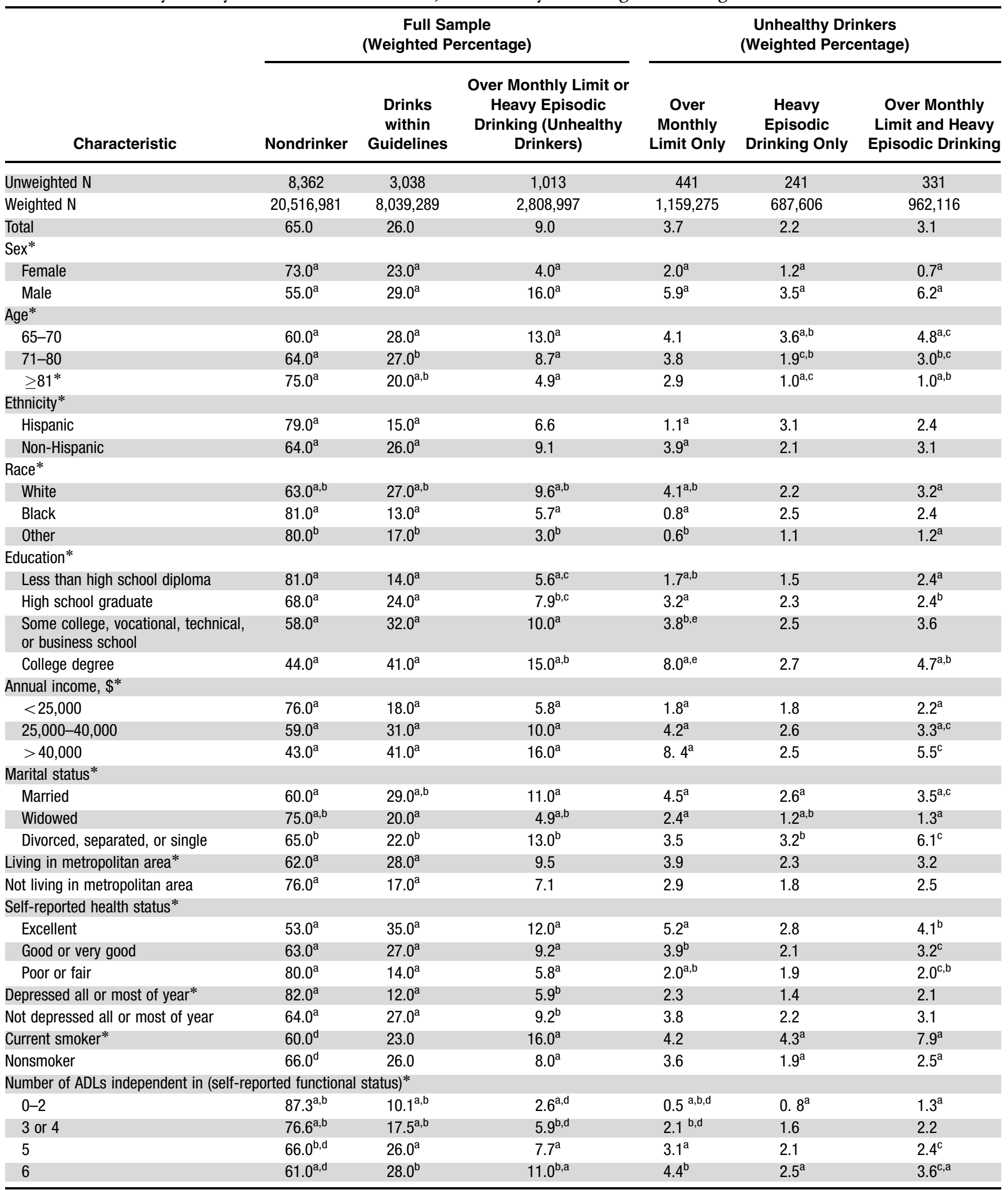

* Global chi-square, $P<.01$.

Note: Pairs that share superscript letter within the same independent variable category and column are significantly different $(\mathrm{a}, \mathrm{b}, \mathrm{d}=P<.01, \mathrm{c}=P<.05)$. Respondents with missing data on alcohol survey items were excluded from the sample ( $n=70,<1 \%$ unweighted of otherwise eligible respondents). Totals may not equal $100 \%$ because of rounding. Over monthly limit is defined as drinking more than 30 drinks per month. Heavy episodic drinking is defined as drinking more than three drinks on any single day in a typical month.

$\mathrm{ADL}=$ activity of daily living. 
prevalence especially pronounced in the over-monthly-limits category $(P<.01)$. Divorced, separated, or single elderly people were more likely than married persons to exceed both limits $(P<.05)$. Better health and functional status showed a monotonic, positive association with unhealthy drinking (global chi-square, $P<.01)$. Current smokers were more likely to be unhealthy drinkers $(P<.01)$ and were especially more likely to be heavy episodic drinkers $(P<.01)$.

\section{Regression Results}

Multivariate logistic regression modeling of any unhealthy drinking (exceeding monthly or single-day limits) in the full sample confirmed many, although not all, of the bivariate associations (Table 3). Higher education, higher income, and reported excellent health were positively associated with unhealthy drinking. Women were less likely to be unhealthy drinkers (odds ratio $(\mathrm{OR})=0.26,95 \%$ confidence interval $(\mathrm{CI})=0.22-0.31$ ), as were older age groups (aged
$71-80, \quad \mathrm{OR}=0.73,95 \% \quad \mathrm{CI}=0.61-0.89 ;$ aged $\geq 81$, $\mathrm{OR}=0.52,95 \% \mathrm{CI}=0.42-0.65)$ and blacks $(\mathrm{OR}=0.60$, $95 \% \mathrm{CI}=0.43-0.84)$ or respondents of other races $(\mathrm{OR}=0.34,95 \% \mathrm{CI}=0.17-0.65)$ than whites. Current smoking in the full sample was associated with more than twice the likelihood of unhealthy drinking $(\mathrm{OR}=2.20$, $95 \% \mathrm{CI}=1.77-2.74)$, although Hispanic ethnicity, being widowed, and self-reported chronic depression were not significant when other characteristics were controlled for. Divorced, separated, or single marital status was associated with a much higher likelihood of unhealthy drinking than being married $(\mathrm{OR}=1.90,95 \% \mathrm{CI}=1.52-2.38)$, which was not the case in bivariate analyses.

When the sample was restricted to drinkers, sex, age, marital status, "other" race, income, and smoking status predicted unhealthy drinking in the same directions as in the population model. Black race, education, and health and functional status variables were not significant here, in contrast to the full sample model and the bivariate analyses.

Table 3. Factors Associated with Any Unhealthy and Heavy Episodic Drinking in Elderly Medicare Beneficiaries: 2003, Logistic Regression Results (Weighted)

Odds Ratio (95\% Confidence Interval)

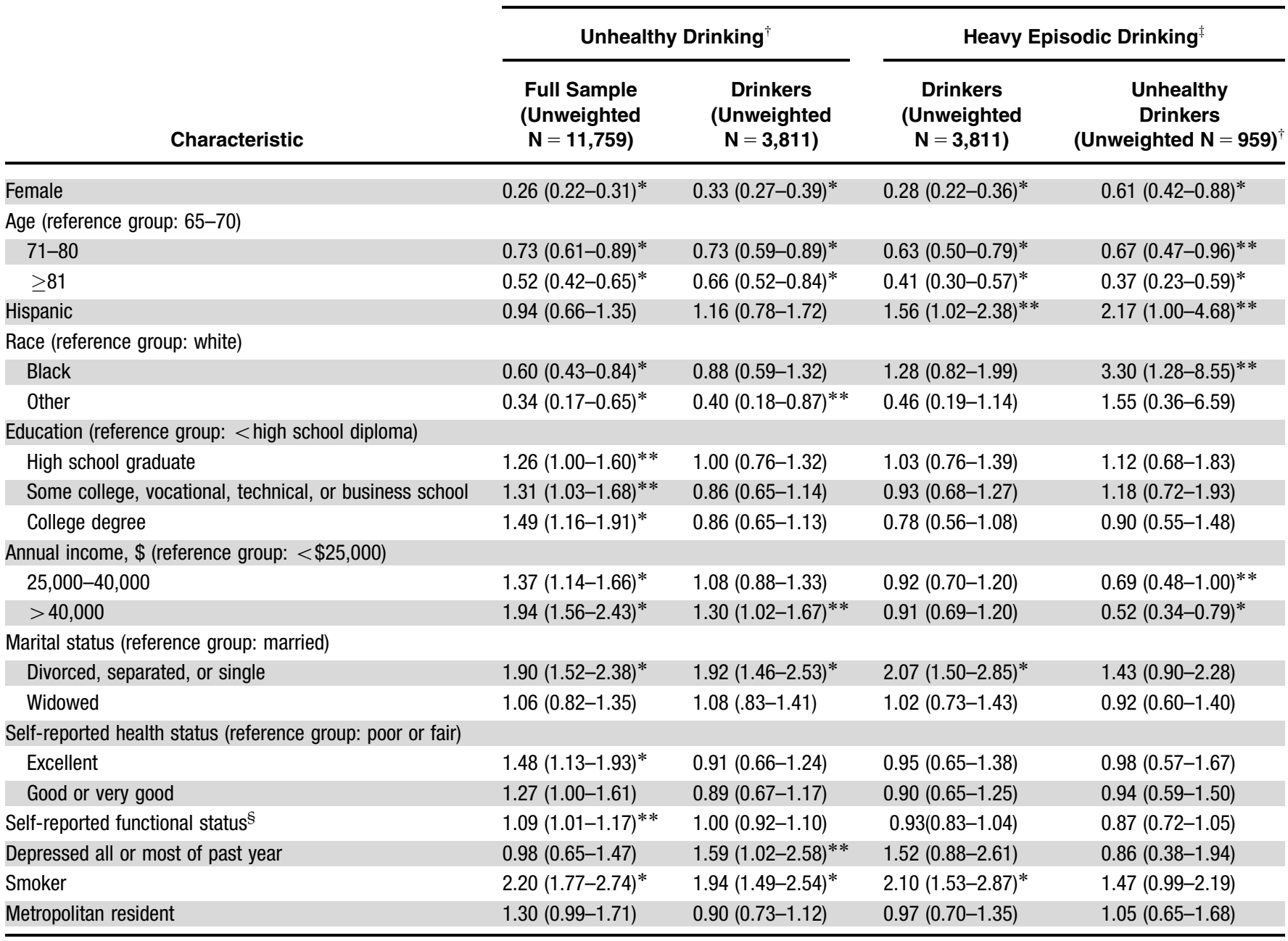

$P<{ }^{*} .01,{ }^{* *} .05$.

${ }^{\S}$ Functional status is measured according to number of self-reported independent activities of daily living (ADLs) on a scale of $0-6$, where 6 represents the highest number of independent ADLs.

${ }^{\dagger}$ Unhealthy drinkers reported consuming more than 30 drinks per month, more than three drinks on any single day in typical month, or both.

${ }^{\ddagger}$ Heavy episodic drinkers reported exceeding the three-drink threshold on any single day in a typical month. 
In this model, self-reported chronic depression was a positive predictor of unhealthy drinking $(\mathrm{OR}=1.59,95 \%$ $\mathrm{CI}=1.02-2.58)$.

Another model predicted heavy episodic drinking (exceeding single-day limit) in the drinker subsample. In drinkers, sex; age; divorced, separated, or single marital status; and current smoking were significant, as in previous analyses. In addition, heavy episodic drinking was significantly more likely for Hispanics $(\mathrm{OR}=1.56$, 95\% CI $=1.02-$ 2.38)

To consider factors associated with heavy episodic drinking from another angle, the model was implemented using the subsample of unhealthy drinkers. In unhealthy drinkers (those who exceeded the 30-drink monthly limit or the single-day heavy episodic threshold), race, and ethnicity were significant factors. Hispanic $(\mathrm{OR}=2.17,95 \%$ $\mathrm{CI}=1.00-4.68)$ and black $(\mathrm{OR}=3.30,95 \% \mathrm{CI}=1.28-$ $8.55)$ unhealthy drinkers were more likely to binge drink than non-Hispanics and whites, respectively.

The final set of logistic regression models analyzed the relationship between respondent characteristics and the likelihood of "dual risk" drinking, defined as exceeding both monthly and single-day limits (Table 4). First this was examined in the subsample of all drinkers. Female sex $(\mathrm{OR}=0.18, \quad 95 \% \quad \mathrm{CI}=0.12-0.27) \quad$ and oldest age $(\mathrm{OR}=0.42,95 \% \mathrm{CI}=0.29-0.61)$ were negatively associated with "dual risk" drinking. Divorced, separated, or single marital status $(\mathrm{OR}=2.57,95 \% \mathrm{CI}=1.69-3.91)$ and current smoking $(\mathrm{OR}=2.45,95 \% \mathrm{CI}=1.76-3.41)$ were strong predictors of "dual risk" alcohol consumption in drinkers. These relationships held-except for marital status - when the model was implemented in the unhealthy drinking subsample.

An ordinary least squares regression model was used to determine predictors of the number of days of heavy episodic drinking in a typical month for the drinker subsample (data not shown). Many of the same variables that predicted any heavy episodic drinking also predicted number of binge days. Being female, being aged 81 and older, and having a college degree predicted fewer heavy episodic drinking days, whereas being divorced, separated, or single or being a current smoker predicted more such drinking

Table 4. Factors Associated with "Dual Risk” Drinking in Elderly Medicare Beneficiaries: 2003, Logistic Regression Results

“Dual Risk” Drinking, Odds Ratio (95\% Confidence Interval)

Characteristic

\section{Female}

Age (reference group: $65-70$ )

$71-80$
$>81$

Race (reference group: white)

Hispanic

Black

Other

Education (reference group: < high school diploma)

High school graduate

Some college, vocational, technical, or business school

College degree

Annual income, $\$$ (reference group: $<\$ 25,000$ )

$25,000-40,000$

$>40,000$

Marital status (reference group: married)

Divorced, separated, or single

Widowed

Self-reported health status (reference group: poor or fair)

Excellent

Good or very good

Self-reported functional status ${ }^{\S}$

Depressed all or most of previous year

Smoker

Metropolitan resident

\section{Drinkers Unhealthy Drinkers}

(Unweighted $\mathrm{N}=\mathbf{3 , 8 1 1}$ )

$0.18(0.12-0.27)^{*} \quad 0.36(0.23-0.56)^{*}$

$0.75(0.55-1.02)$

$0.42(0.29-0.61)^{*}$

$0.91(0.56-1.49)$

$0.84(0.48-1.47)$

$0.47(0.15-1.44)$

$0.84(0.56-1.27)$

$0.88(0.61-1.28)$

$0.68(0.45-1.03)$

$1.00(0.73-1.37)$

$1.25(0.87-1.80)$

\subsection{7 (1.69-3.91)*}

$1.11(0.75-1.64)$

$1.16(0.74-1.83)$

$1.13(0.76-1.67)$

$0.90(0.77-1.04)$

$1.54(0.78-3.04)$

$2.45(1.76-3.41)^{*}$

$1.03(0.70-1.50)$
$0.98(0.68-1.43)$

$0.53(0.34-0.84)^{*}$

$0.71(0.37-1.35)$

$0.93(0.44-1.94)$

$1.07(0.25-4.60)$

$0.84(0.52-1.35)$

$1.00(0.61-1.62)$

$0.72(0.44-1.17)$

$0.91(0.63-1.33)$

$1.03(0.66-1.60)$

1.67 (0.99-2.81)

0.99 (0.65-1.52)

$1.29(0.73-2.26)$

$1.33(0.83-2.12)$

$0.90(0.74-1.10)$

$1.05(0.50-2.24)$

$1.87(1.31-2.66)^{*}$

$1.17(0.75-1.81)$

${ }^{*} P<.01$.

$\dagger$ "Dual risk" drinkers report more than 30 drinks monthly and heavy episodic drinking.

${ }^{\ddagger}$ Unhealthy drinkers report more than 30 drinks monthly or heavy episodic drinking.

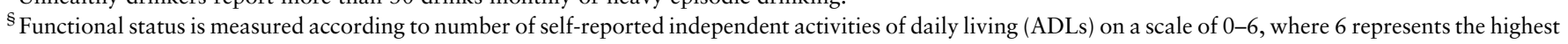
number of independent ADLs. 
days, although in this model, age of 71 to 80 and Hispanic ethnicity variables were no longer significant. "Other" race and having a college degree became significant negative predictors, and there was a significant inverse relationship with functional status.

\section{DISCUSSION}

This study found that two-thirds of elderly Medicare beneficiaries do not report drinking, one-quarter drink within recommended guidelines, and a significant minority report alcohol consumption patterns that exceed guidelines. Approximately $9 \%$ exceed the monthly 30 -drink limit or the single-day limit of three drinks. Prevalence was sharply higher for men $(16 \%)$ and for those aged 65 to $70(13 \%)$. The unhealthy drinking prevalence reported here is consistent with some prior reports, although somewhat higher than others. As mentioned previously, definitions of heavy, problem, or unhealthy drinkers in prior research vary. In following the NIAAA and AGS guidelines, a relatively low consumption threshold was set. In addition, parameters of drinking guidelines (heavy episodic drinking (exceeding single-day or occasion threshold) as well as typical daily or weekly (extrapolated in the data from monthly) consumption) were included.

The results revealed that a significant proportion $(>40 \%)$ of those who exceeded guidelines did so through exceeding the monthly limit only. This finding is in contrast to that in the general population of at-risk drinkers aged 21 and older, of whom approximately $92 \%$ who exceed guidelines exceed single-day limits. ${ }^{34}$ Because the large majority of this group reported drinking two drinks per day-an acceptable level for men younger than 65 -it is possible that many of this unhealthy drinking subgroup may be particularly amenable to education or advice. Some portion of them may simply be continuing drinking patterns that were not risky when they were younger, unaware of their heightened age-related risk. It will be important to investigate this type of drinker further, particularly using longitudinal data. In addition, although the NIAAA ${ }^{8}$ currently recommends the use of a single screening question about heavy drinking days and studies validate its use in primary care, ${ }^{35,36}$ this approach may be less sensitive in this population than strategies that ask about typical daily amounts, such as the earlier NIAAA recommendations and other screening instruments that have been validated for use in an elderly population. ${ }^{1,37-40}$

Many of the factors found to be associated on a bivariate basis with unhealthy drinking in elderly people confirm prior research. Higher income was associated with unhealthy drinking, as was better functional and self-perceived health status. Men; younger people; those with higher levels of education and income; smokers; whites; and divorced, single, or separated elderly people were more likely to drink unhealthy amounts. A lower prevalence of unhealthy drinking was found in those reporting depression, a point on which previous studies had found varying relationships.

The bivariate examination of specific drinking patterns also revealed some important new information. For example, whites were particularly more likely to drink over monthly limits only. Hispanics were less likely to do so. This type of result suggests the possibility of customizing interventions to fit drinking patterns that may be differentially prevalent in subpopulations. An intervention for older individuals drinking, for example, two drinks per day, because they assumed it was good for them might differ in emphasis from an intervention for someone who has heavy drinking episodes several times a month.

Multivariate analyses with different samples also yielded some valuable information. It is useful to model unhealthy drinking, and specific categories thereof, with both population and drinker samples. For example, although Hispanic ethnicity was not significantly related to unhealthy drinking overall, among unhealthy drinkers, it was positively associated with heavy episodic drinking. When considering the full population, health status is positively related to the likelihood of unhealthy drinking, although when the sample was restricted to drinkers, health status was no longer a predictor. Conversely, subsample analysis revealed that, although self-reported chronic depressive symptomatology was not related to unhealthy drinking in the full population, it was a significant positive predictor of unhealthy drinking among drinkers. In addition, subjects with depression were less likely to be unhealthy drinkers, but when other factors were controlled for, the associational direction reversed. Findings regarding depression and functional status as they relate to unhealthy drinking bear further investigation.

The findings from the study underscore the value of continuing to develop and use broad and targeted approaches to reducing alcohol misuse. Understanding factors associated with unhealthy drinking in the general population of older individuals helps guide broad public awareness efforts. Noting factors associated with unhealthy drinking in self-reported drinkers can heighten professional attention in health care and other service settings, a critical need given the widespread underrecognition of alcohol problems. Furthermore, differences between drinkers can inform low-risk drinking recommendations by identifying factors associated with unhealthy use. For example, older unhealthy drinkers with heavy episodic drinking may represent different likelihoods of formal alcohol disorders from the likelihoods of those who continue to drink at levels that were acceptable for earlier phases of life, and these two groups may be responsive to different approaches. Various subpopulations-defined along dimensions including sex, race, and ethnicity - may tend toward distinct unhealthy drinking patterns best addressed through individualizing messages and interventions in new ways. Although universal screening is currently recommended, in practice, screening may be more selective. Data identifying subgroups at higher risk may help clinicians in that regard. Furthermore, attention to those at greater risk may lead to more frequent screening rather than, for instance, a single screening for new patients or at an annual examination.

Interventions developed in response to identified unhealthy drinking patterns and risk factors must also be shaped according to a more-in-depth understanding of the specific contexts and characteristics of the drinking behaviors. For example, if "happy hours" in some assisted living facilities and other settings for older adults play a significant role in unhealthy drinking for certain subgroups, then education and intervention may be important in those 
settings. At the individual level, understanding the unique history and meaning of these drinking patterns - including how they may be intertwined with meeting social and emotional needs - is critical.

Furthermore, it may be that widely publicized messages regarding benefits of moderate drinking are obscuring the more-nuanced realities. Some evidence suggests that many older adults define moderate alcohol use at levels above what is recommended by guidelines. ${ }^{41}$ Also, moderate drinking benefits accrue differentially to people with various demographic and health characteristics. Helping individuals to be aware of the risks and benefits of alcohol consumption in the context of their own situation is an important goal.

The study has some limitations. Some individuals may underreport alcohol consumption. Although self-reported alcohol consumption is in general considered to be as accurate as other measures of drinking, ${ }^{42}$ elderly people may be more likely to underreport alcohol use because of stigma. ${ }^{1,39}$ In addition, because of certain disease states and interactions between medication and alcohol not captured here, a portion of the group that drinks within guidelines is likely to be using alcohol in circumstances that place them at risk. ${ }^{43}$ For these reasons, even though the low recommended consumption thresholds were used, the prevalence of unhealthy drinking found is a conservative estimate based upon alcohol guidelines in general. In interpreting these results, it is critical to recognize that the relationships found between drinking patterns and sociodemographic, health status, and other variables are associations; with these data, it is not possible to determine causality. Information was not available on past drinking or changes in alcohol consumption over time. Also, the prevalence of formal alcohol disorders could not be ascertained from the data.

The findings of the study are valuable in providing current, generalizable information regarding the prevalence of - and factors associated with - various patterns of unhealthy drinking in elderly, community-dwelling Medicare beneficiaries. Further research is needed to examine implications for clinical and health education interventions for these drinking patterns in older adults.

\section{ACKNOWLEDGMENTS}

The authors thank Grant Ritter, PhD, for statistical consultation, Marian Ryan for research assistance, and Michele Hutcheon for manuscript preparation. We have listed everyone who contributed significantly to this manuscript, and we have received written consent from all contributors who are not authors and are acknowledged above.

Financial Disclosure: This study was funded by $\mathrm{Na}-$ tional Institute on Alcohol Abuse and Alcoholism Grant 5R21AA015746. None of the authors report a conflict of interest.

Author Contribution: Elizabeth Merrick, Constance Horgan, Deborah Garnick, Dominic Hodgkin, Richard Saitz, and Frederic Blow participated in developing the study concept, research design, and analytical approach; interpreting data; and developing the manuscript (writing or giving substantive input). Lee Panas contributed to developing and implementing the analytical approach, conducted statistical programming, participated in interpreting data, and gave input on the manuscript. Susan Houghton contributed to developing the analytical approach, interpreting data, and drafting the manuscript.

Sponsor's Role: The role of the sponsor was limited to funding the project as proposed.

\section{REFERENCES}

1. Blow F. Substance Abuse Among Older Adults: Treatment Improvement Procotol (TIP) Series 26. Rockville, MD: SAMHSA, 1998

2. Saitz R. Unhealthy alcohol use. N Engl J Med 2005;352:596-607.

3. Adams WL, Yuan Z, Barboriak J et al. Alcohol-related hospitalizations of elderly people. JAMA 1993;270:1222-1225.

4. Saitz R. Medical and surgical complications of addiction. In: Graham A, Schultz T, Mayo-Smith M, Ries R, Wilford B, eds. Principles of Addiction Medicine, 3rd Ed. Chevy Chase, MD: American Society of Addiction Medicine, 2003, pp 1027-1052.

5. Mukamal KJ, Chung H, Jenny NS et al. Alcohol consumption and risk of coronary heart disease in older adults: The cardiovascular health study. J Am Geriatr Soc 2006;54:30-37.

6. Abramson JL, Williams SA, Krumholz HM et al. Moderate alcohol consumption and risk of heart failure among older persons. JAMA 2001;285:20042006.

7. Mukamal KR, Kuller LH, Fitzpatrick AL et al. Prospective study of alcohol consumption and risk of dementia in older adults. JAMA 2003;289:14051413.

8. Helping Patients Who Drink Too Much. A Clinician's Guide 2005 Edition. Rockville, MD: NIAAA, National Institutes of Health, 2005.

9. Moore A. American Geriatrics Society Clinical Practice Committee. Clinical Guidelines for Alcohol Use Disorders in Older Adults. November 2003, Clinical Guideline [on-line]. Available at http://www.americangeriatrics.org/ products/positionpapers/alcohol.shtml Accessed September 20, 2006.

10. Moos R, Brennan P, Schutte K et al. High-risk alcohol consumption and late life alcohol use problems. Am J Public Health 2004;94:1985-1991.

11. Blow F, Barry KL, Fuller B et al. Analysis of the National Health and Nutrition Examination Survey (NHANES): Longitudinal analysis of drinking over the life span. In: Korper SP, Council CL, eds. Substance Use by Older Adults: Estimates of Future Impact on the Treatment System (DHHS Publication No. SMA 03-3763, Analytic Series A-21). Rockville, MD: SAMHSA, 2002, pp 125-141.

12. Moore A, Giuli L, Gould R et al. Alcohol use, comorbidity and mortality. J Am Geriatr Soc 2006;54:757-762.

13. Fleming MF. Identification and treatment of alcohol use disorders in older adults. In: Gurnack AM, Atkinson R, Osgood N, eds. Treating Alcohol and Drug Abuse in the Elderly. New York: Springer, 2002, pp 85-108.

14. Breslow R, Faden V, Smothers B. Alcohol consumption by elderly Americans. J Stud Alcohol 2003;11:884-892.

15. National Survey on Drug Use and Health, Substance Abuse and Mental Health Services Administration [on-line]. Available at http://www.oas.samhsa.gov/ NSDUH/2k5NSDUH/2k5results.htm\#Ch3 Accessed May 1, 2007.

16. Serdula MK, Brewer RD, Gillespie $C$ et al. Trends in alcohol use and binge drinking, 1985-1999. Am J Prev Med 2004;26:294-298.

17. Kirchner JE, Zubritsky C, Cody $\mathrm{M}$ et al. Alcohol consumption among older adults in primary care. J Gen Intern Med 2007;22:92-97.

18. Oslin D, Holden R. Recognition and assessment of alcohol and drug dependence in the elderly. In: Gurnack AM, Atkinson R, Osgood N, eds. Treating Alcohol and Drug Abuse in the Elderly. New York: Springer, 2002, p. 242.

19. Grant BF, Dawson DA, Stinson FS et al. The 12-month prevalence and trends in DSM-IV alcohol abuse and dependence: United States, 1991-1992 and 2001-2002. Drug Alcohol Depend 2004;74:223-234.

20. Hajat S, Haines A, Bulpitt C et al. Patterns and determinants of alcohol consumption in people aged 75 years and older: Results from the MRC trial of assessment and management of older people in the community. Age Ageing 2004;33:170-177.

21. Ganry O, Joly J-P, Queval M-P et al. Prevalence of alcohol problems among elderly patients in a university hospital. Addiction 2002;95:107-113.

22. Brennan P, Moos R. Late-life drinking behavior: The influence of personal characteristics, life context and treatment. Alcohol Health Res World 1996;20:197-204.

23. Benshoff J, Harrawood L. Substance abuse and the elderly: Unique issues and concerns. J Rehabil 2003;69:43-48.

24. Blow F. Special issues in treatment: Older adults. In: Graham A, Schultz T, Mayo-Smith M, Ries R, Wilford B, eds. Principles of Addiction Medicine, 3rd Ed. Chevy Chase, MD: American Society of Addiction Medicine, 2003, pp 581-607. 
25. Cassidy K, Kotynia-English R, Acres J et al. Association between lifestyle factors and mental health measures among community-dwelling older women. Aust NZ J Psychiatry 2004;38:940-947.

26. Saunders P, Copeland J, Dewey M et al. Heavy drinking as a risk factor for depression and dementia in elderly men: Findings from the Liverpool Longitudinal Community Study. Br J Psychiatry 1991;159:213-216.

27. Blow F, Oslin D, Barry KL. Misuse and abuse of alcohol, illicit drugs, and psychoactive medication among older people. Generations 2002;26:50-54.

28. Moore RD, Bone LR, Geller G et al. Prevalence, detection and treatment of alcoholism in hospitalized patients. JAMA 1989;261:403-407.

29. Centers for Medicare and Medicaid. Medicare Current Beneficiary Survey Overview [on-line]. Available at http://www.cms.hhs.gov/apps/mcbs/Overview. asp Accessed October 3, 2006

30. Centers for Medicare and Medicaid. Medicare Current Beneficiary Survey Main Study - Round 37-Fall Supplement 2003 Community Component HF. Health Status and Functioning [on-line]. Available at http://www.cms.hhs. gov?MCBS/downloads/2003_CBQ_hs.pdf Accessed October 10, 2007.

31. Wallace M, Shelkey M. Katz Index of Independence in Activities of Daily Living. Best Practices in Nursing Care to Older Adults. The Hartford Institute for Geriatric Nursing, 1998.

32. Cohen SB. An evaluation of alternative PC-based software packages developed for the analysis of complex survey data. Am Stat 1997;51: 285-292.

33. Lemeshow S, Letenneur L, Dartigues JF et al. Illustration of analysis taking into account complex survey considerations: The association between wine consumption and dementia in the PAQUID study. Am J Epidemiol 1998; 148:298-306.

34. Dawson DA. US low-risk drinking guidelines: An examination of four alternatives. Alcohol Clin Exp Res 2000;24:1820-1829.

35. Williams R, Vinson D. Validation of a single screening question for problem drinking. J Fam Pract 2001;50:307-312.

36. Seale JP, Boltri JM, Shellenberger S et al. Primary care validation of a single screening question for drinkers. J Stud Alcohol 2006;67:778-784.

37. Carrington Reid M, Tinetti ME, O’Connor PG et al. Measuring alcohol consumption among older adults: A comparison of available methods. Am J Addict 2003;12:211-219.

38. Adams WL, Barry KL, Fleming MF. Screening for problem drinking in older primary care patients. JAMA 1996;276:1964-1967.

39. Wilcox S, King A. Alcohol consumption in older adults: A comparison of two assessment methods. J Appl Gerontol 2000;19:170-181.

40. Fink A, Morton S, Beck JC et al. The Alcohol-Related Problems Survey: Identifying hazardous and harmful drinking in older primary care patients. J Am Geriatr Soc 2002;50:1717-1722.

41. Masters JA. Moderate alcohol consumption and unappreciated risk for alcohol-related harm among ethnically diverse urban dwelling elders. Geriatr Nurs 2004;24:155-161.

42. Babor TM, Steinberg K, Anton R et al. Talk is cheap: Measuring drinking outcomes in clinical trials. J Stud Alcohol 2000;61:55-63.

43. Moore A, Morton S, Beck JC et al. A new paradigm for alcohol use in older persons. Med Care 1999;37:165-179. 